\title{
Efficient Modal Analysis of Periodic Structures Loaded With Arbitrarily Shaped Waveguides
}

\author{
Stephan Marini, Ángela Coves, Member, IEEE, Vicente E. Boria, Senior Member, IEEE, and \\ Benito Gimeno, Member, IEEE
}

\begin{abstract}
In this paper, a novel full-wave method for the modal characterization of closed metallic periodic structures based on arbitrarily shaped waveguides is presented. This method relies on an integral equation formulation solved via the method of moments, which finally leads to the solution of a standard eigenvalue problem. The required modal spectrum of waveguides with arbitrary cross section is determined through the boundary integral-resonant mode expansion technique. For validation purposes, the proposed analysis method is first successfully applied to standard waveguide periodic geometries already considered in the technical literature. Our new algorithm is then used to identify the higher order Floquet modes, as well as to compute the related Brillouin diagrams, of complex closed metallic periodic structures loaded with arbitrarily shaped waveguides.
\end{abstract}

Index Terms-Arbitrarily shaped waveguides, complex modes, dispersion diagram, electromagnetic bandgap, Floquet modes, periodic structures.

\section{INTRODUCTION}

$\mathbf{R}$ ECENTLY, a renewed interest in periodic waveguide components is being observed, which can be attributed to the many interesting properties and practical applications of electromagnetic and photonic bandgap structures [1]. Periodically loaded waveguides can be considered the fundamental key component of such structures, and their propagation characteristics play an important role in the design procedure of many microwave devices such as slow-wave structures, resonators and filters, backward-wave oscillators, impedance-matching devices, phase shifters, polarizers, corrugated antennas, antenna feeds, and accelerating structures [2]-[10]. Therefore, there is an increasing request of accurate and efficient analysis tools that can cope with more complex periodic structures [11], [12].

Manuscript received January 07, 2008; revised December 01, 2009. First published February 08, 2010; current version published March 12, 2010. This work was supported by the Ministerio de Educación y Ciencia, Spanish Government, under coordinated Research Project TEC 2007/67630-C03-01.

S. Marini is with the Departamento de Física, Ingeniería de Sistemas y Teoría de la Señal, Universidad de Alicante, E-03690 Alicante, Spain (e-mail: smarini@dfists.ua.es).

Á. Coves is with the Departamento de Física y Arquitectura de Computadores, Universidad Miguel Hernández de Elche, 03202 Elche, Alicante, Spain (e-mail: angela.coves@umh.es).

V.E. Boria is with the Departamento de Comunicaciones, Instituto de Telecomunicaciones y Aplicaciones Multimedia, Universidad Politécnica de Valencia, 46022 Valencia, Spain (e-mail: vboria@dcom.upv.es).

B. Gimeno is with the Departamento de Física Aplicada, Instituto de Ciencia de los Materiales, Universidad de Valencia, 46100 Burjasot, Valencia, Spain (e-mail: benito.gimeno@uv.es).

Digital Object Identifier 10.1109/TMTT.2010.2040407
In the past, several analytical and numerical methods have been proposed in order to characterize periodic structures, which can be essentially grouped into two main categories. The first group consists of methods that determine the propagation constants, following an iterative procedure, as the roots of a nonlinear determinant equation [8]. This approach can be time consuming and can miss degenerate or nearly degenerate roots, thus introducing an additional difficulty to identify complex modes. Such drawbacks disappear in the methods of the second group, where the propagation constants are obtained from the solution of a generalized eigenvalue matrix problem, which is derived using either a modal expansion [3], a generalized scattering matrix representation [13], or the finite-element method [14]. Other techniques, either based on artificial transmission lines or following a Bloch wave analysis method, have been successfully used for characterizing the dispersive behavior of periodic planar transmission lines [15]-[17]. The accuracy of these methods has been recently improved through the use of a full-wave analysis method based on a periodic layered-medium Green's function [18].

Most of the closed metallic periodic waveguide structures considered in the technical literature are based on standard rectangular and circular waveguides, either corrugated [19], [20] or periodically loaded with standard geometry irises [21]. Recently, the guided-wave characteristics of rectangular waveguide based periodic structures, loaded with transversal strip layers (i.e., with the shape of square loops or crosses), have been solved for the fundamental mode (see [22]). In this paper, we will consider more complex closed metallic periodic structures composed of the cascaded connection of arbitrarily shaped waveguides, whose cross sections can be defined by any combination of linear, circular, and/or elliptical arcs, as shown in Fig. 1(a).

In order to solve the proposed arbitrary geometries, we will make use of a rigorous and efficient method based on the integral-equation analysis technique described in [23]. The application of this technique requires to know the modal chart of all arbitrarily shaped waveguide sections involved in the periodic structure under consideration. For this purpose, the well-known boundary integral-resonant mode expansion method, originally proposed in [24] and recently revisited in [25], is employed. After solving the waveguide steps and uniform sections involved in a single cell of the periodic structure, a standard eigenvalue problem is formulated in terms of the transmission $(A B C D)$ chain matrix, whose solution provides the propagation constants of the fundamental and higher order Floquet modes. A method based on the correlation between eigenvectors at two different 


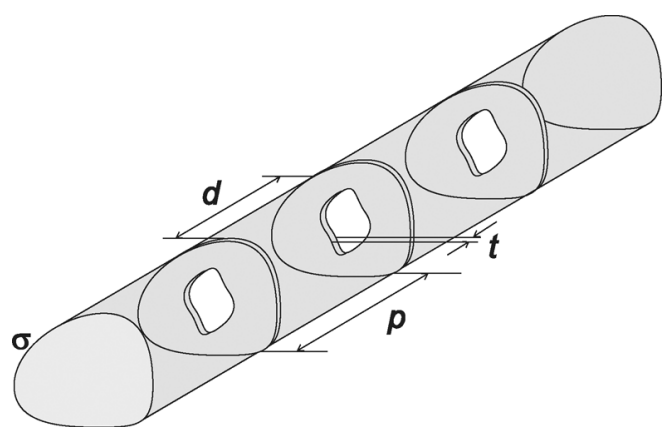

(a)

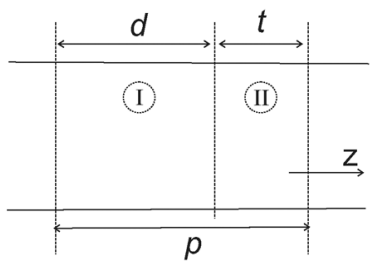

(b)

Fig. 1. Periodic structure loaded with: (a) arbitrarily shaped waveguides in and (b) side view of the unit cell.

frequencies is used to trace the dispersion diagram, thus allowing to identify multimode regions and the proper sorting of supported propagating, evanescent and complex modes.

To fully validate the accuracy of the method proposed in this paper, we have first applied it to analyze three periodic structures already considered in previous publications: two of them are standard corrugated rectangular and circular waveguides, and the last one is based on a rectangular waveguide periodically loaded with symmetrical metal inserts (ridges). Our novel characterization method has then been used to compute the dispersion diagrams of more complex closed periodic structures based on arbitrarily shaped waveguides. In particular, a rectangular waveguide periodically loaded with cross-shaped irises, and a circular waveguide with periodic elliptical irises, have been studied. The propagation results for both structures have also been validated through comparisons with the magnitude of the transmission coefficient for the same truncated topologies (i.e., considering only a finite number of unit cells).

\section{THEORY}

In this section, we will present the theoretical basics related to the proposed analysis technique. As shown in Fig. 1(a), the periodic structure under consideration consists of the cascade connection of arbitrarily shaped waveguides, whose cross section $\sigma$ is defined by any combination of linear, circular, and/or elliptical arcs. The unit cell of length $p$ is composed of two regions [see Fig. 1(b)], where $d$ represents the length of the groove region, and $t=p-d$ is the thickness of the iris.

The $A B C D$ chain matrix of one period $p$ of the structure under study $(\mathbf{M})$ is directly obtained by solving the cascade connection of two arbitrary waveguides

$$
\mathbf{M}=\left[\begin{array}{ll}
\mathbf{A} & \mathbf{B} \\
\mathbf{C} & \mathbf{D}
\end{array}\right]=\prod_{i=1}^{4}\left[\begin{array}{ll}
\mathbf{A}_{\mathbf{i}} & \mathbf{B}_{\mathbf{i}} \\
\mathbf{C}_{\mathbf{i}} & \mathbf{D}_{\mathbf{i}}
\end{array}\right]
$$

where the coefficients of the first and third $A B C D$ matrices are trivial since they correspond to hollow waveguides with finite lengths (i.e., the first one is the host waveguide, whereas the other one is formed by the interior of the loading aperture). The second and fourth matrices are related to the modeling of the same planar waveguide junction.

In order to obtain the full-wave characterization of the involved planar junction, a very efficient method based on an integral-equation technique [23] is followed. The final aim of this method is to provide an accurate full-wave modal representation of a waveguide junction in terms of a generalized impedance matrix. An important contribution of the method is the distinction made between accessible and localized modes: accessible modes are those used to connect adjacent transitions, while localized modes are only used to describe the electromagnetic fields close to the junction (the number of localized modes is usually considerably greater than the number of accessible modes, both determined to get convergent results).

The application of this efficient full-wave analysis method requires the knowledge of the modal charts related to the waveguides of the planar junction under consideration, as well as the coupling coefficients between the modes of these waveguides. To obtain this information, the well-known boundary integral-resonant mode expansion technique, originally proposed in [24], is employed. More details about the extension of such technique for the rigorous consideration of arbitrary cross sections, defined by any combination of linear, circular, and/or elliptical segments, can be found in [25].

Once the $2 \times 2$ blocks generalized $Z$-matrix of the planar waveguide junction is determined, the corresponding transmission $(A B C D)$-parameters are derived as follows:

$$
\begin{aligned}
& A=Z_{11} \cdot Z_{21}^{-1} \\
& B=Z_{11} \cdot Z_{21}^{-1} \cdot Z_{22}-Z_{12} \\
& C=Z_{21}^{-1} \\
& D=Z_{21}^{-1} \cdot Z_{22}
\end{aligned}
$$

and then the transmission matrix $\mathbf{M}$ can be easily computed using (1). We must now recall that in a periodic structure of period $p$, any component $F(z)$ of the electromagnetic fields must satisfy the Floquet condition [3]

$$
F(z+p)=e^{-\gamma p} F(z) \quad \forall z
$$

where $\gamma$ is the propagation constant of the modes of the infinite periodic structure. Imposing the Floquet condition at the unit cell, we obtain

$$
\left(\begin{array}{c}
\mathbf{V}_{1} \\
\mathbf{I}_{1}
\end{array}\right)=\mathbf{M}\left(\begin{array}{c}
\mathbf{V}_{2} \\
-\mathbf{I}_{2}
\end{array}\right)=e^{\gamma p}\left(\begin{array}{c}
\mathbf{V}_{2} \\
-\mathbf{I}_{2}
\end{array}\right)
$$

The right-hand side of (7) can be expressed in the classical canonical form 
where $\mathbf{I}$ is the identity matrix. The solutions ( $\boldsymbol{\Lambda}$ and $\mathbf{x}$ ) of the standard eigenvalue problem, which are related respectively to the required propagation constants and transverse field distributions in the periodic structure, can be straightforwardly determined using well-established routines.

At a given frequency, the real and imaginary parts of the propagation constant, i.e., $\gamma=\alpha+j \beta$, corresponding to a Floquet mode are directly related to the vector component $\Lambda_{i}$ through the following relationships:

$$
\alpha_{i}=\frac{\ln \left|\Lambda_{i}\right|}{p} \quad \beta_{i}=\frac{\angle \Lambda_{i}}{p} .
$$

When solving the canonical problem defined by (8), the number of eigenvalue solutions $\Lambda$ is equal to the order of the matrix $\mathbf{M}$ in the left-hand side. These eigenvalues are, in general, complex and appear as pairs corresponding to forward and backward directions of propagation of the same mode. Passbands and stopbands are characterized, respectively, by the condition as to whether or not the periodic structure supports at least one propagating Floquet mode within the frequency range considered. Clearly, passbands occur when the Floquet mode propagates, and the corresponding pair of eigenvalues has a phase propagator with $\left|\Lambda_{i}\right|=1$. However, if a Floquet mode is evanescent, its pair of eigenvalues moves to a value higher than $1\left|\Lambda_{i}\right|>1$ (corresponding to the solution evanescent in the $+z$-direction) and to a value $\left|\Lambda_{i}\right|<1$ (corresponding to the solution evanescent in the $-z$-direction) [26].

In conclusion, the nontrivial solutions of the problem and the resulting points of the dispersion diagram are solved frequency by frequency. In some cases, for instance, when several modes are simultaneously excited (multimode region), we have noticed that the solutions are mixed up since the eigenvalues at different frequencies do not appear in the same order. To overcome this problem, we have followed a method based on computing the correlation between eigenvectors at two different frequency points. In [27], this method was originally proposed for ordering the solutions of a nonlinear determinant equation, related to the characterization of planar electromagnetic-bandgap structures.

This correlation-based method computes by means of (7), for any subinterval $\left[f_{a}, f_{b}\right]$ of the frequency band under analysis, the eigenvalue vectors $\boldsymbol{\Lambda}_{\mathrm{a}}$ and $\boldsymbol{\Lambda}_{\mathrm{b}}$ and the corresponding eigenvector matrices $\mathbf{V}_{\mathbf{a}}$ and $\mathbf{V}_{\mathbf{b}}$ at $f_{a}$ and $f_{b}$, respectively. The eigenvalues have to be arranged in ascending order of amplitude, and the eigenvectors are orthogonal and normalized according to

$$
\mathbf{V}_{\mathbf{a}}^{\mathbf{T}} \mathbf{V}_{\mathbf{a}}^{*}=\mathbf{I} \quad \mathbf{V}_{\mathrm{b}}^{\mathbf{T}} \mathbf{V}_{\mathrm{b}}^{*}=\mathbf{I}
$$

where $\mathbf{T}$ denotes the transpose and $*$ denotes the complex conjugate.

In order to find the correct sorting of the solutions, a correspondence between eigenvalue vectors $\boldsymbol{\Lambda}_{\mathrm{a}}$ and $\boldsymbol{\Lambda}_{\mathrm{b}}$ is needed. This is obtained by calculating a correlation matrix between the corresponding eigenvectors

$$
\mathbf{R}=\mathbf{V}_{\mathbf{a}}^{\mathbf{T}} \mathbf{V}_{\mathbf{b}}^{*}
$$

Due to the normalization condition (10), if the $i$ th eigenvector at frequency $f_{a}$ slightly differs from the $j$ th eigenvector at frequency $f_{b}$, the element $R_{i j}$ is close to unity. Thus, there is a strong correlation between $\Lambda_{a i}$ and $\Lambda_{b j}$. Conversely, $R_{i j}$ is close to zero when there is no correlation between the two eigenvalues. It is important to note that the eigenvalues are ordered so the correlation matrix $\mathbf{R}$ usually exhibits unity elements in the main diagonal. If the position of two corresponding eigenvalues are different at $f_{a}$ and $f_{b}$, the matrix $\mathbf{R}$ exhibits elements close to unity out of the main diagonal. A threshold procedure is applied to $R_{i j}$ elements in order to obtain a new matrix where in any row or column only appears one element equal to unity, and all other elements are zero. This procedure detects crossings between eigenvalues, and therefore, allows the correct computation of the dispersion curve in a frequency range considering all interactions among higher order modes.

The previous procedure is slightly modified to deal with some special cases. In particular, when the unit cell supports $M$ degenerate modes, $M$ columns and $M$ rows of the matrix $\mathbf{R}$ exhibit more than one element different from both 0 and 1 since the eigenvectors are mixed up. However, in this case, these elements are easily identified, and a Gram-Schmidt orthogonalization procedure is applied to make the eigenvectors linearly independent. Another problem typically arises when the frequency interval is chosen too large, and the eigenvectors are weakly correlated, thus different elements of the matrix $\mathbf{R}$ differ from 0 or 1. In this case, a simple solution is to reduce the frequency interval and then to apply the same procedure. For all the examples considered in this paper, we have chosen a frequency interval smaller than $0.3 \%$ of the central frequency band under analysis, which has always provided successful results. It is important to note that the two previous problematic cases are easily detected and solved in a fully automated way.

Finally, we must say that the computational effort related to the whole procedure described for finding the dispersion diagram of periodic waveguide structures is rather low. On the one hand, the canonical eigenvalue problem outlined in (8) is quickly solved since the dimension of the $\mathbf{M}$ matrix (directly related to the number of accessible modes chosen in the waveguides) is typically small. On the other side, the modal analysis of arbitrarily shaped waveguides is frequency independent so it is performed only once and outside the frequency loop.

\section{RESULTS}

Next, we proceed to study the accuracy and efficiency of the proposed analysis technique through several examples. First, we have computed the dispersion diagram and behavior of three periodically loaded structures based on standard rectangular and circular waveguides. In particular, a corrugated rectangular waveguide, a rectangular waveguide periodically loaded with symmetrical $E$-plane metal ridges, and a corrugated circular waveguide have been considered, and their results successfully verified through comparison with data available in the technical literature. Secondly, we have applied our theory to solve a rectangular waveguide periodically loaded with cross-shaped irises, and to a circular waveguide including periodic elliptical insertions. For these two cases, we have compared our results with the simulated transmission $\left(S_{21}\right)$ parameters of the same 


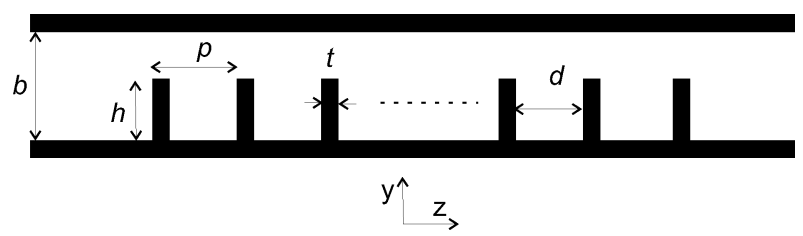

Fig. 2. Layout and dimensions of a rectangular waveguide periodically loaded with asymmetrical capacitive irises. $a=22.86 \mathrm{~mm}, b=5.08 \mathrm{~mm}, h=$ $3.81 \mathrm{~mm}, p=10 \mathrm{~mm}$, and $t=0.001 \mathrm{~mm}$.

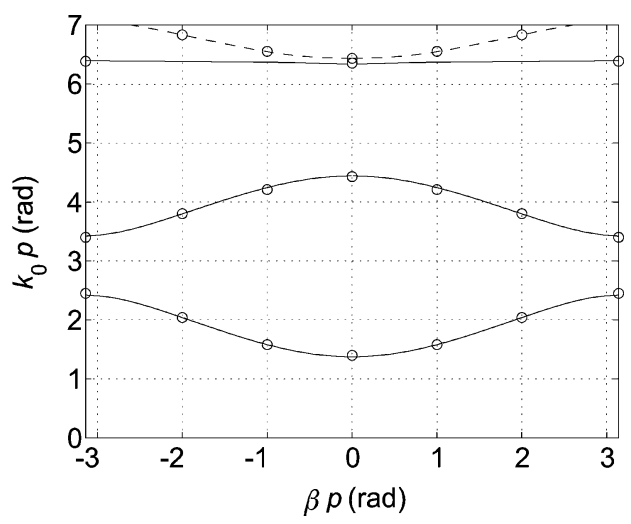

Fig. 3. $k_{0}-\beta$ diagram of the corrugated rectangular waveguide shown in Fig. 2. The circles are from [21]. The solid line corresponds to the first Floquet propagating mode and the dashed line corresponds to the second one.

structures considering finite lengths. All the reported computational efforts have been obtained with an Intel Core 2 platform at $2.4 \mathrm{GHz}$ with 1-GB RAM.

\section{A. Validation Examples}

First of all, in order to validate our theory and the developed computer code, we have considered a rectangular waveguide periodically loaded with very thin asymmetrical capacitive irises (see the geometry in Fig. 2).

This classical example (with zero thickness irises) was first investigated by Collin [3], and successively by Amari et al. including higher order modes [21]. For the analysis of this structure, we have considered $\mathrm{TE}_{1 n}^{z}$ and $\mathrm{TM}_{1 n}^{z}$ modes, as well as infinitely thin irises $(t=0.001 \mathrm{~mm})$. Fig. 3 represents the $k_{0}-\beta$ diagram, when the unit cell has a period of $p=10 \mathrm{~mm}$, the rectangular waveguide has a size of $a=22.86 \mathrm{~mm}$ and $b=5.08 \mathrm{~mm}$, and the insertion of the irises is $h=3.81 \mathrm{~mm}$. The results predicted by our method (with solid and dashed lines) are successfully compared with numerical data from [21] (with circles). Passbands and stopbands are clearly evident. The solid line corresponds to the first Floquet mode, and the dashed line corresponds to the second one, which have been clearly distinguished by our analysis technique.

Fig. 4 shows the real and imaginary parts of the propagation constants of the first two modes as a function of frequency for the same corrugated rectangular waveguide. In this figure, the modes with $\alpha \neq 0$ represent evanescent solutions. The points, between $9.95 \leq k_{0} p \leq 10.14$, indicate a pair of complex modes, which were also detected by Amari et al. [21] and firstly suggested by Cooper [28]. As was explained in [26], the emergence of complex modes in this kind of structures can be identified by the presence of cutoff transitions at $\beta p \neq(0, \pm \pi)$. From

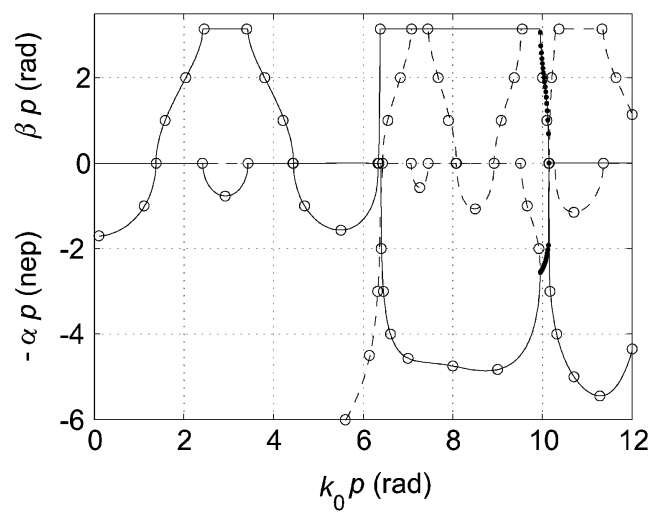

Fig. 4. Dispersion diagram for the corrugated rectangular waveguide described in Fig. 2. The solid line corresponds to the first Floquet propagating mode and the dashed line to the second one. The points show a pair of complex modes and the circles are from [21].

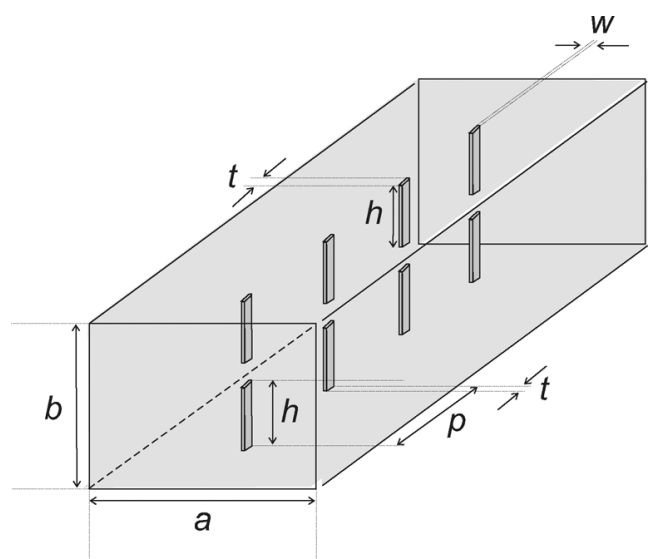

Fig. 5. Layout of a WR-90 waveguide periodically loaded with symmetrical ridges. The dimensions are $a=22.86 \mathrm{~mm}, b=10.16 \mathrm{~mm}, w=0.1 \mathrm{~mm}$ $h=4.58 \mathrm{~mm}, p=8 \mathrm{~mm}$, and metal thickness $t=2 \mathrm{~mm}$.

Fig. 4, we can conclude that our results are in excellent agreement with those obtained from [21] (circles).

The next example deals with the analysis of a WR-90 waveguide periodically loaded with symmetrical metal insertions, which has been proposed in [29] for designing bandpass $E$-plane filters with improved stopband response. The layout of this periodic structure is presented in Fig. 5, where the symmetrical ridges placed in the upper and lower walls have a width and height of $w=0.1 \mathrm{~mm}$ and $h=4.58 \mathrm{~mm}$, respectively.

In Fig. 6, we present the Brillouin diagram of the structure with period $p=8 \mathrm{~mm}$ and metal thickness for the ridges $t=2 \mathrm{~mm}$. The solid line corresponds to the first Floquet mode and the dashed line to the second one. A very good agreement between our results (displayed with solid and dashed lines) and those reported in [29] (circles) can be observed. However, it must be noted that the multimode region shown between 23.5-25 GHz was not included in [29]. In fact, according to our results, the two Floquet modes join together at $24.88 \mathrm{GHz}$, and then generate a pair of complex modes (which represent an evanescent field).

Exploiting the symmetries of this structure, our analysis method has required to consider 50 accessible modes, 150 basis functions, and 500 kernel terms for solving the involved 


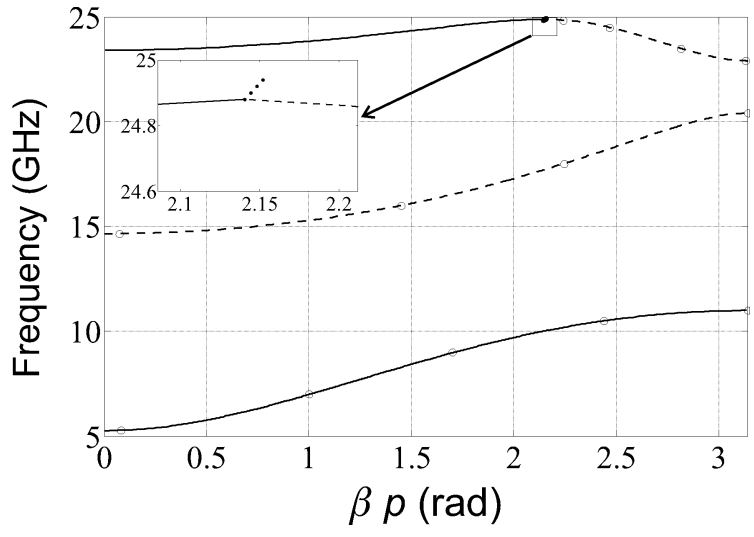

Fig. 6. Brillouin diagram for a WR-90 waveguide periodically loaded with two symmetrical ridges, as shown in Fig. 5. The solid line corresponds to the first Floquet mode, and the dashed line to the second one. The circles are taken from [29].

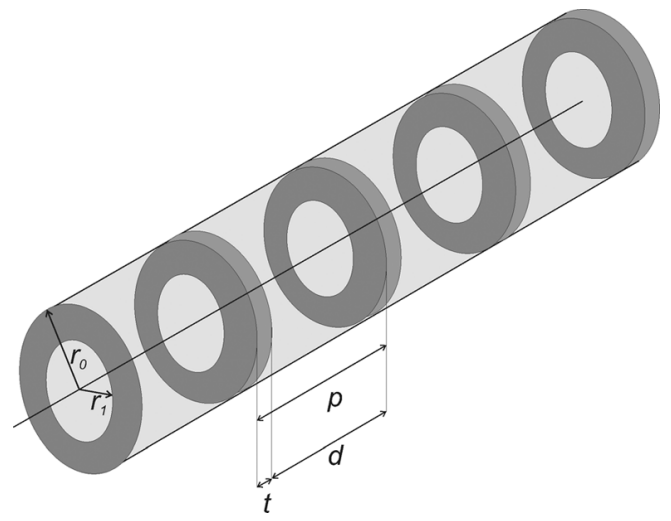

Fig. 7. Layout of a corrugated circular waveguide. The dimensions are $r_{1}=$ $10 \mathrm{~mm}, r_{1} / r_{0}=0.4, p=9 \mathrm{~mm}$, and $t=4 \mathrm{~mm}$.

planar junctions. In this case, the complete analysis technique, including also the computation of the dispersion diagram and considering the first six modes for solving (8), has only taken a computational effort of $0.016 \mathrm{~s}$ per frequency value (i.e., $16 \mathrm{~s}$ for 1000 frequency points). Such numerical effort is very well compared to the one required by available commercial software tools that can cope with this kind of ridged structures.

Once the new proposed theory has been successfully validated with periodic structures based on rectangular waveguides, we have performed a further verification considering a corrugated circular waveguide (see topology in Fig. 7). In particular, this kind of corrugated structures has also been analyzed with other methods by Amari et al. [21], as well as by Esteban and Rebollar [19]. For the particular example considered in this paper (Fig. 7), we have computed the corresponding dispersion curve considering the circular waveguide modes with unity angular dependence (only $\mathrm{TE}_{1 n}^{z}$ and $\mathrm{TM}_{1 n}^{z}$ modes) since these are the relevant ones for the study of corrugated antennas and antenna feeds [8].

In Fig. 8 , we can see the $k_{0}-\beta$ diagram for the corrugated circular waveguide described in Fig. 7. The first four Floquet modes provided by our technique are represented with different lines, whereas the circles indicate numerical data taken from

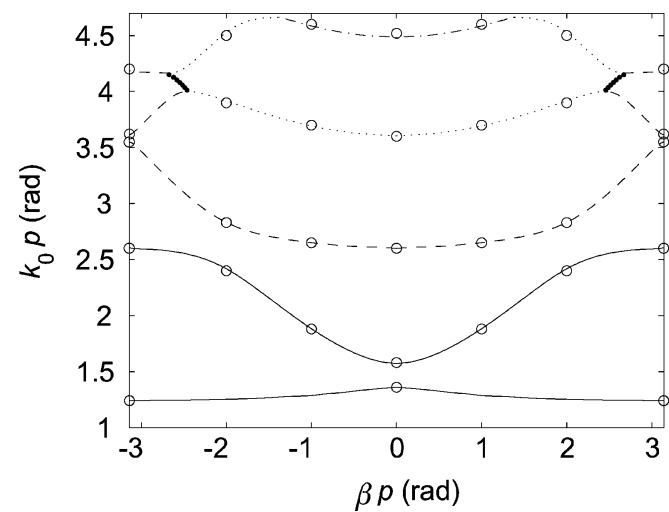

Fig. 8. $k_{0}-\beta$ diagram for the first four Floquet modes of the corrugated circular waveguide shown in Fig. 7. The solid lines correspond to the first mode, the dashed lines represent the second one, whereas the dotted and the dashed-dotted lines represent the third and fourth ones, respectively. The circles are taken from [21], and the points represent a pair of complex modes.

[21]. As can be observed, an excellent agreement between both results is obtained. The first passbands are present for $1.24 \leq$ $k_{0} p \leq 1.35$ and for $1.57 \leq k_{0} p \leq 2.59$, and between them a first bandgap appears. These bands are related to the first Floquet mode (solid line in Fig. 8). Two additional passbands are also present for $2.6 \leq k_{0} p \leq 3.55$ and $3.61 \leq k_{0} p \leq 4.0$, where a multimode region (two or more Floquet modes are propagating) is observed (see dashed and dotted lines in Fig. 8 defining the second passband). Another multimode region is also found for $4.16 \leq k_{0} p \leq 4.66$, where the passband is made up of three Floquet modes (see the dashed, dotted, and dashed-dotted lines in Fig. 8). A pair of complex modes, represented with points in Fig. 8, can be observed for $4.0 \leq k_{0} p \leq 4.16$ (note that these modes represent a nonpropagative field). Finally, as it was also pointed out in [21], the group velocity of the lowest mode $\left(1.24 \leq k_{0} p \leq 1.35\right)$ is negative so this first branch is supporting backward waves.

In order to find the correct solution for this corrugated circular waveguide, an intensive use of the proposed sorting technique based on the correlation matrix has been required since the first four solutions (Floquet modes) of (7) are mixed up in the considered frequency band. For the first time, to the authors' knowledge, the application of such technique has allowed the identification of the first four Floquet modes clearly shown in Fig. 8. Such results have been obtained using 150 accessible modes, 200 basis functions, and 500 kernel terms in the integral equations related to the solution of each planar junction. Using the first seven $\mathrm{TE} / \mathrm{TM}_{1 n}^{z}$ modes, we have then solved the matrix equation defined in (8). All these numerical operations have involved a total computational effort of $0.65 \mathrm{~s}$ per frequency point (500 points).

\section{B. Periodic Structures Loaded With Arbitrarily Shaped Waveguides}

Next, we will consider more complex periodic structures based on rectangular and circular waveguides loaded with arbitrarily shaped waveguides. For instance, the first example consists of a rectangular waveguide periodically loaded with 


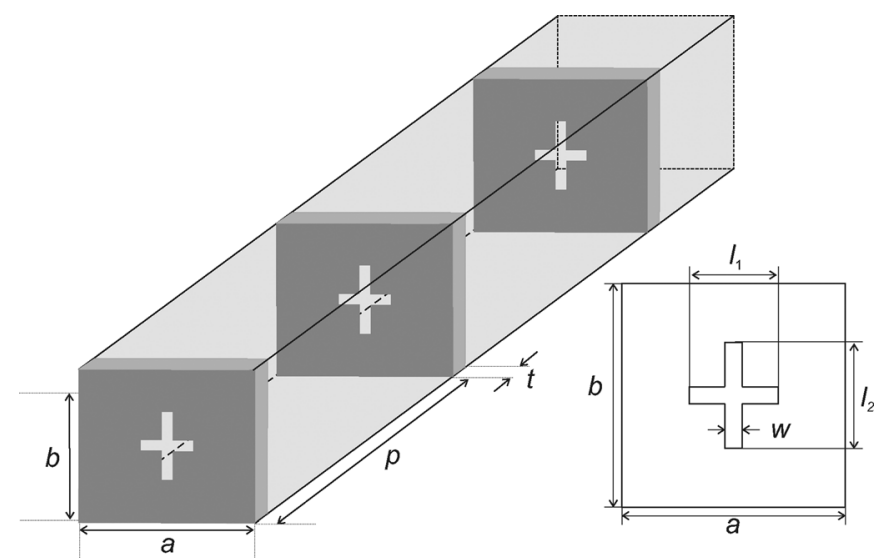

Fig. 9. Layout of the rectangular waveguide periodically loaded with centered cross-shaped irises. $a=22.86 \mathrm{~mm}, b=20 \mathrm{~mm} ; w=3 \mathrm{~mm}, l_{1}=15.3 \mathrm{~mm}$ and $l_{2}=17.3 \mathrm{~mm} ; p=11 \mathrm{~mm}$ and thickness $t=1 \mathrm{~mm}$.

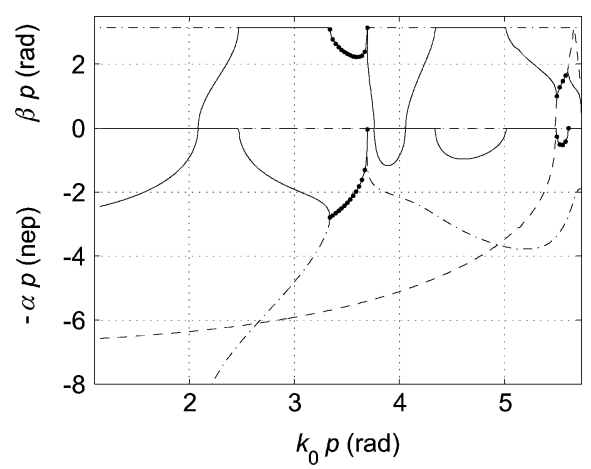

Fig. 10. Dispersion response for a rectangular waveguide periodically loaded with centered cross-shaped irises shown in Fig. 9. Solid line: first Floquet mode. Dashed (second) and dashed-dotted lines: other higher order Floquet modes. The points represent a pair of complex modes.

centered cross-shaped irises, whose geometry and dimensions can be seen in Fig. 9.

Fig. 10 shows the real and imaginary parts of the normalized propagation constant as a function of $k_{0} p$ for the first three Floquet modes of the structure shown in Fig. 9. For the full-wave analysis of such a periodic structure, only $\mathrm{TE} / \mathrm{TM}_{m n}^{z}$ modes with $m$ odd and $n$ even have been considered. As can be seen, there is no propagation until $k_{0} p=2.09$, where the first Floquet mode appears (solid line). Two passbands are present in the ranges defined by $2.09 \leq k_{0} p \leq 2.47$ and $3.69 \leq k_{0} p \leq 3.75$, and there is a stopband between them, where the first Floquet mode is evanescent $\left(2.47 \leq k_{0} p \leq 3.33\right)$ and a pair of complex modes represented with points is present $\left(3.33 \leq k_{0} p \leq 3.69\right)$. The first Floquet mode also propagates in the ranges of frequencies $4.05 \leq k_{0} p \leq 4.34$ and $5.0 \leq k_{0} p \leq 5.49$. On the other hand, at $k_{0} p=5.49$ a new Floquet mode (denoted via the dashed line) appears, contributing to form a pair of complex modes in $5.49 \leq k_{0} p \leq 5.6$. Finally, for $k_{0} p \geq 5.6$, a multimode region is present because the first two Floquet modes are propagating simultaneously. The third Floquet mode, represented via the dashed-dotted line, is an evanescent mode practically in all the frequency range, except for $3.33 \leq k_{0} p \leq 3.69$, where it contributes to form the first pair of complex modes.

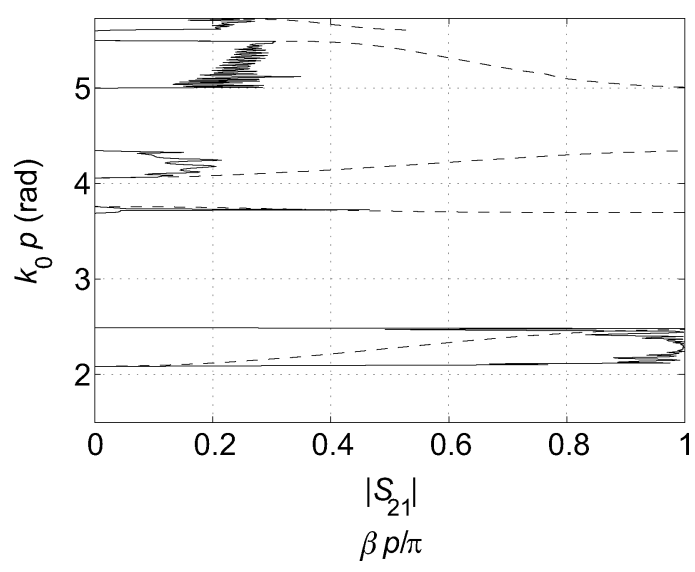

Fig. 11. Brillouin diagram of the first Floquet mode (dashed line) and module of the transmission coefficient (solid line) of the periodic structure detailed in Fig. 9 considering a finite number of unit cells ( 40 periods).

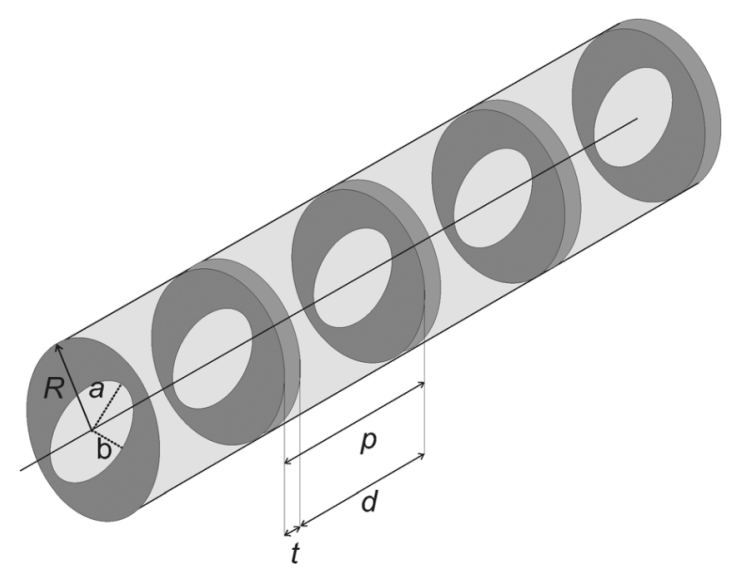

Fig. 12. Layout of the circular waveguide periodically loaded with centered elliptical irises. $R=5 \mathrm{~mm} ; a=3.5 \mathrm{~mm}, b=2.87 \mathrm{~mm} ; p=6 \mathrm{~mm}$ and thickness $t=2 \mathrm{~mm}$.

The previous results were obtained using 80 accessible modes, 100 basis functions, and 500 kernel terms for solving the planar waveguide junctions of the structure. In order to compute the displayed dispersion diagram, the first six TE/TM $m n$ (with $m$ odd and $n$ even) modes have been used. The complete analysis procedure has required a global computational effort of $0.6 \mathrm{~s}$ per frequency (500 points in total).

With the aim of verifying the accuracy of the previous results, Fig. 11 successfully compares the $\beta-k_{0}$ diagram for the first Floquet mode of the periodic rectangular waveguide with the transmission coefficient for the fundamental mode $\left(\mathrm{TE}_{10}^{z}\right)$ of the same structure, but considering a finite number of unit cells (40 periods).

The last example considered in this study deals with a circular waveguide structure periodically loaded with elliptical irises since the use of elliptical waveguides has generated great interest in high-power applications and in vacuum electronics due to low space-charge energy and efficient coupling to RF structures [10]. The layout and dimensions of the periodic structure under analysis is shown in Fig. 12, whereas the real and imaginary parts of the normalized propagation constant as 


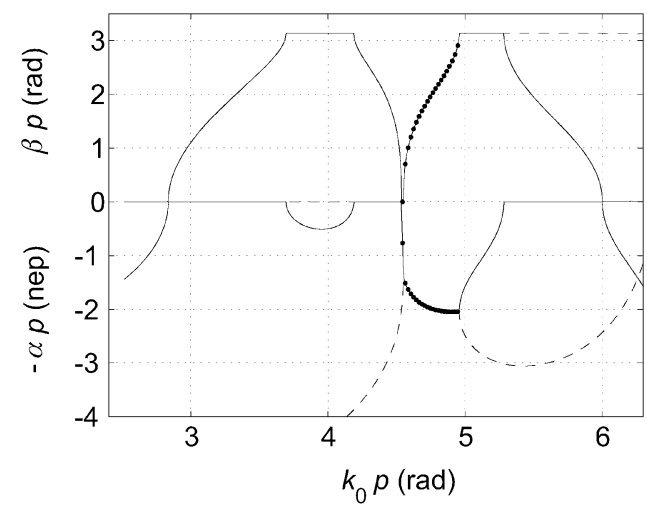

Fig. 13. Dispersion response for a circular waveguide periodically loaded with centered elliptical irises shown in Fig. 12. Solid line: first Floquet mode. Dashed line: the second Floquet mode. The points represent a pair of complex modes.

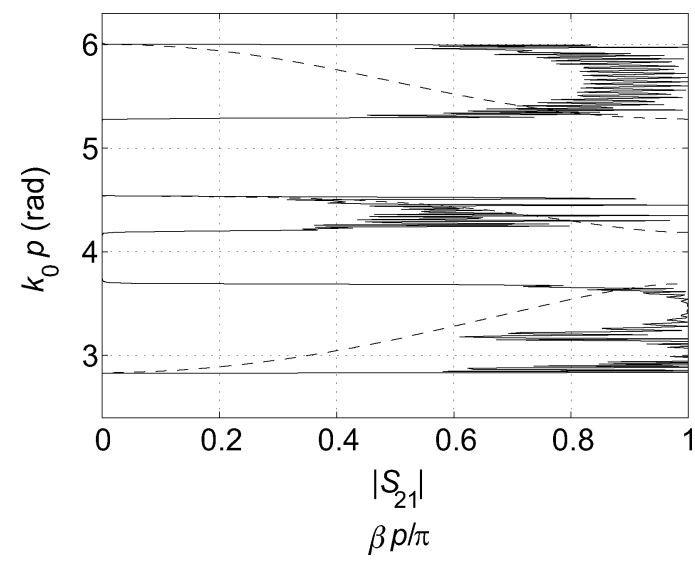

Fig. 14. Brillouin diagram of the first Floquet mode (dashed line) and module of the transmission coefficient (solid line) of the periodic structure shown in Fig. 12 considering a finite number of unit cells ( 30 periods).

a function of frequency for the first two Floquet modes of such structure are displayed in Fig. 13.

From Fig. 13, it is concluded that passbands are present for $2.83 \leq k_{0} p \leq 3.70,4.19 \leq k_{0} p \leq 4.53$ and $5.28 \leq k_{0} p \leq 6.0$, where the first Floquet mode (solid line) propagates. The points at $4.54 \leq k_{0} p \leq 4.97$ represent a pair of complex modes, and the second Floquet mode (dashed line) is evanescent in the remaining frequency range. In order to validate these results, we have successfully compared again (see Fig. 14) the propagation constant of the first Floquet mode of this structure with the magnitude of the transmission coefficient for the fundamental mode of the same structure, but including only a finite number of unit cells (30 periods). The minimum of transmission, which appears at $k_{0} p \sim \pi$ (where only the first Floquet mode propagates), is caused by the fundamental TE mode of the elliptical waveguide iris that has a cutoff frequency of $f=25.278 \mathrm{GHz}$.

Our results were obtained using, in this case, 150 accessible modes, 200 basis functions, and 400 kernel terms for applying the integral equation to all involved planar junctions. Considering only the first four $\mathrm{TE} / \mathrm{TM}_{m n}^{z}$ modes (with $m$ odd) in the circular and elliptical waveguides, we have then computed the dispersion diagram of this periodic structure solving the eigenvalue problem defined by (8). The global computational effort needed by our technique has been equal to $0.39 \mathrm{~s}$ per frequency value (1000 points in total), which clearly validates the numerical efficiency of the proposed method for solving complex closed metallic periodic waveguide structures.

\section{CONCLUSION}

A very efficient tool for the full-wave modal characterization of closed metallic periodic structures, based on the cascade connection of arbitrarily shaped waveguides, has been described. This tool allows the accurate computation of the dispersion diagram (related to the fundamental and higher order Floquet modes) of the cited periodic structures, where the cross section of the arbitrary waveguides can be defined by any combination of linear, circular, and/or elliptical arcs. The novel proposed analysis tool is based on the combination of an integral-equation technique and the well-known boundary integral-resonant mode expansion method, thus leading to the final solution of a generalized eigenvalue matrix problem. For validation purposes, such method has been first applied to geometries already considered in the technical literature such as corrugated rectangular and circular waveguides, as well as rectangular waveguides loaded with metal ridges in the $E$-plane. Finally, the dispersion diagrams of more complex closed metallic periodic structures have been successfully determined. For instance, propagating, evanescent, and complex modes of a rectangular waveguide periodically loaded with cross-shaped irises, and of a periodic structure based on circular and elliptical waveguides, have been properly identified, sorted, and computed. In the last two examples, the dispersion behavior of the fundamental Floquet mode has been compared to the transmission parameter of the same truncated topologies.

\section{REFERENCES}

[1] S. G. Johnson and J. D. Joannopoulus, Photonic Crystals: The Road From Theory to Practice. Boston, MA: Kluwer, 2002.

[2] L. Brillouin, Wave Propagation in Periodic Structure. New York: Dover, 1953.

[3] R. E. Collin, Field Theory of Guided Waves. New York: McGrawHill, 1960.

[4] P. J. B. Clarricoats and M. I. Sobhy, "Propagation behavior of periodically loaded waveguides," Proc. Inst. Elect. Eng., vol. 115, pp. 652-661, 1968.

[5] G. H. Bryant, "Propagation in corrugated waveguides," Proc. Inst. Elect. Eng., vol. 116, pp. 203-213, 1969.

[6] P. J. B. Clarricoats and P. K. Saha, "Propagation and radiation behavior of corrugated feeds," Proc. Inst. Elect. Eng., vol. 118, pp. 1167-1176, Sep. 1971.

[7] M. S. Navarro, T. E. Rozzi, and Y. T. Lo, "Propagation in a rectangular waveguide periodically loaded with resonant irises," IEEE Trans. Microw. Theory Tech., vol. MTT-28, no. 8, pp. 857-865, Aug. 1980.

[8] P. J. B. Clarricoats and A. D. Olver, Corrugated Horns for Microwave Antennas. Stevenage, U.K.: Peregrinus, 1984.

[9] A. D. Olver, P. J. B. Clarricoats, A. A. Kishk, and L. Shafai, Microwave Horns and Feeds. Piscataway, NJ: IEEE Press, 1994.

[10] R. Bhatt, T. Bemis, and C. Chen, "Three-dimensional theory and simulation of non relativistic elliptic electron and ion beam generation," IEEE Trans. Plasma Sci., vol. 34, no. 2, pp. 187-193, Apr. 2006.

[11] C. M. Soukoulis, Photonic Band Gap Structure. Norwell, MA Kluwer, 1996.

[12] J. D. Joannopoulos, R. D. Meade, and J. N. Winn, Photonic Band Gap Structure. Princeton, NJ: Princeton Univ. Press, 1995.

[13] R. Lech and J. Mazur, "Propagation in rectangular waveguide periodically loaded with cylindrical posts," IEEE Microw. Wireless Compon. Lett., vol. 14, no. 4, pp. 177-179, Apr. 2004

[14] T. Itoh, G. Pelosi, and P. P. Silvester, Finite Element Software for Microwave Engineering. New York: Wiley, 1996. 
[15] S.-G. Mao and M.-Y. Chen, "Propagation characteristics of finite-width conductor-backed coplanar waveguides with periodic electromagnetic bandgap cells," IEEE Trans. Microw. Theory Tech., vol. 50, no. 11, pp. 2624-2628, Nov. 2002.

[16] L. Zhu, "Guided-wave characteristics of periodic coplanar waveguides with inductive loading-unit-length transmission parameters," IEEE Trans. Microw. Theory Tech., vol. 51, no. 10, pp. 2133-2138, Oct. 2003.

[17] J. Gao and L. Zhu, "Characterization of infinite- and finite-extent coplanar waveguide metamaterials with varied left- and right-handed passbands," IEEE Microw. Wireless Compon. Lett., vol. 15, no. 11, pp. 805-807, Nov. 2005.

[18] S. Paulotto, P. Baccarelli, F. Frezza, and D. R. Jackson, "Full-wave modal dispersion analysis and broadside optimization for a class of microstrip CRLH leaky-wave antennas," IEEE Trans. Microw. Theory Tech., vol. 56, no. 12, pp. 2826-2837, Dec. 2008.

[19] J. Esteban and M. Rebollar, "Characterization of corrugated waveguides by modal analysis," IEEE Trans. Microw. Theory Tech., vol. 39 , no. 6, pp. 937-943, Jun. 1991.

[20] S. Amari, R. Vahldieck, and J. Bornemann, "Analysis of propagation in periodically loaded circular waveguides," Proc. Inst. Elect. Eng.-Microw. Antennas Propag., vol. 146, no. 1, pp. 50-54, Feb. 1999.

[21] S. Amari, R. Vahldieck, J. Bornemann, and P. Leuchtmann, "Spectrum of corrugated and periodically loaded waveguides from classical matrix eigenvalues," IEEE Trans. Microw. Theory Tech., vol. 48, no. 3, pp. 453-459, Mar. 2000.

[22] R. S. Kshetrimayum and L. Zhu, "Guided-wave characteristics of waveguide based periodic structures loaded with various FSS strip layers," IEEE Trans. Antennas Propag., vol. 53, no. 1, pp. 120-124, Jan. 2005

[23] G. Gerini, M. Guglielmi, and G. Lastoria, "Efficient integral equation formulations for admittance or impedance representation of planar waveguide junctions," in IEEE MTT-S Int. Microw. Symp. Dig., Jun. 1998, vol. 3, pp. 1747-1750.

[24] G. Conciauro, M. Bressan, and C. Zuffada, "Waveguide modes via an integral equation leading to a linear matrix eigenvalue problem," IEEE Trans. Microw. Theory Tech., vol. MTT-32, no. 11, pp. 1495-1504, Nov. 1984.

[25] S. Cogollos, S. Marini, V. E. Boria, P. Soto, A. Vidal, H. Esteban, J. V. Morro, and B. Gimeno, "Efficient modal analysis of arbitrarily shaped waveguides composed of linear, circular and elliptical arcs using the BI-RME method," IEEE Trans. Microw. Theory Tech., vol. 51, no. 12, pp. 2378-2390, Dec. 2003.

[26] S. Simsek and E. Topuz, "Some properties of generalized scattering matrix representations for metallic waveguides with periodic dielectric loading," IEEE Trans. Microw. Theory Tech., vol. 55, no. 11, pp. 2336-2344, Nov. 2007.

[27] M. Bozzi, L. Germani, S. Minelli, L. Perregrini, and P. de Maagt, "Efficient calculation of the dispersion diagram of planar electromagnetic band-gap structures by the MoM/BI-RME method," IEEE Trans. Antennas Propag., vol. 53, no. 1, pp. 29-35, Jan. 2005.

[28] D. Cooper, "Complex propagation coefficients and the step discontinuity in corrugated cylindrical waveguide," Electron. Lett., vol. 7, pp. 135-136, Mar. 1971.

[29] G. Goussetis, A. Feresidis, and P. Kosmas, "Efficient analysis, design, and filter applications of EBG waveguide with periodic resonant loads," IEEE Trans. Microw. Theory Tech., vol. 54, no. 11, pp. 3885-3892, Nov. 2006.

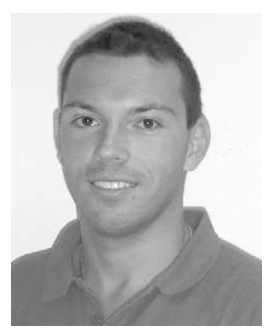

Stephan Marini was born in Cagli, Italy, on January 3, 1976. He received the Laurea degree in electronics engineering from the University of Perugia, Perugia, Italy, in 2001, and the Ph.D. degree in telecommunications from the Universidad Politécnica de Valencia, Valencia, Spain, in 2005.

In June 2001, he joined the Departamento de Comunicaciones, Universidad Politécnica de Valencia, in the frame of the European Union project "Millimeter-wave and Microwave Components Design Framework for Ground and Space Multimedia Network" (MMCODEF). Since 2005, he has been a Lecturer with the Departamento de Física, Ingeniería de Sistemas y Teoría de la Señal, Univer- sidad de Alicante, Alicante, Spain. In 2009, he received the José Castillejo Fellowship from the Spanish Government for a short stay (four months) with the LEMA Laboratory, École Polytechnique Fédérale de Lausanne, Lausanne, Switzerland. His research interests include numerical methods for the analysis of arbitrary-shaped waveguide, scattering, and periodic structures.

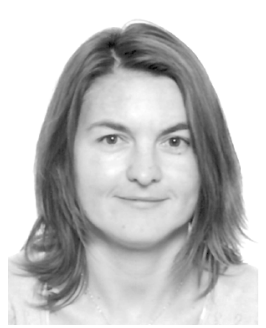

Ángela Coves (S'04-M'05) was born in Elche (Alicante), Spain, on May 20, 1976. She received the Licenciado degree in physics and Ph.D. degree from the Universidad de Valencia, Valencia, Spain, in 1999 and 2004, respectively.

Since 2001, she has been a Lecturer with the Signal Theory and Communications Division, Universidad Miguel Hernández (UMH) de Elche, Elche, Alicante, Spain. Her current research interests include the study of electron discharges in microwave components and computer-aided techniques of analysis of microwave passive components, waveguide structures, and diffraction gratings.

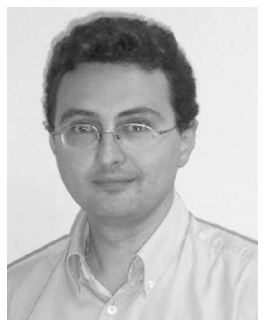

Vicente E. Boria (S'91-A'99-SM'02) was born in Valencia, Spain, on May 18, 1970. He received the Ingeniero de Telecomunicación degree (with first-class honors) and Doctor Ingeniero de Telecomunicación degree from the Universidad Politécnica de Valencia, Valencia, Spain, in 1993 and 1997, respectively.

In 1993, he joined the Departamento de Comunicaciones, Universidad Politécnica de Valencia, where since 2003, he has been a Full Professor. In 1995 and 1996, he was a Spanish Trainee with the European Space Research and Technology Centre (ESTEC)-European Space Agency (ESA), Noordwijk, The Netherlands. He has authored or coauthored five chapters in technical textbooks, 60 papers in refereed international technical journals, and over 150 papers in international conference proceedings. His current research interests are focused on the analysis and automated design of passive components, left-handed and periodic structures, as well as the simulation and measurement of power effects (multipactor and corona) in passive waveguide systems.

Dr. Boria has been a member of the IEEE Microwave Theory and Techniques Society (IEEE MTT-S) and the IEEE Antennas and Propagation Society (IEEE AP-S) since 1992. He is member of the Editorial Boards of the IEEE TRANSACTIONS ON MicRowaVe THEORY AND TECHNIQUES and IEEE MicrowAVE AND WiRELESS COMPONENTS LETTERS. He is also a member of the Technical Committees of the IEEE MTT-S International Microwave Symposium (IMS) and the European Microwave Conference.

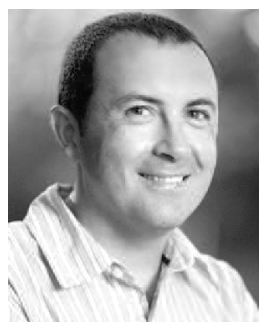

Benito Gimeno (M'01) was born in Valencia, Spain, on January 29, 1964. He received the Licenciado degree in physics and Ph.D. degree from the Universidad de Valencia, Valencia, Spain in 1987 and 1992, respectively.

From 1987 to 1990 , he was a Fellow with the Universidad de Valencia. Since 1990, he has been an Assistant Professor with the Departamento de Física Aplicada y Electromagnetismo, Universidad de Valencia, where, in 1997, he became an Associate Professor. In 1994 and 1995, he was a Research Fellow with the European Space Research and Technology Centre (ESTEC), European Space Agency (ESA). In 2003, he obtained a fellowship from the Spanish Government for a short stay (three months) with the Università degli Studi di Pavia, Pavia, Italy, as a Visiting Scientist. His current research interests include the areas of computer-aided techniques for the analysis of passive components for space applications, waveguides and cavities including dielectric objects, electromagnetic bandgap structures, frequency-selective surfaces, and nonlinear phenomena appearing in power microwave subsystems (multipactor and corona effects). 